\title{
Multiple Vehicles for a Semantic Navigation Across Hyper-environments
}

\author{
Irene Celino and Emanuele Della Valle \\ CEFRIEL - Politecnico of Milano, \\ Via Fucini 2, 20133 Milano, Italy \\ celino@cefriel.it, dellavalle@cefriel.it
}

\begin{abstract}
The Web, but also for example a large extra-net such as a digital library, has an intricate topology that makes navigation through resources a tricky task. The mere introduction of Semantic Web technologies won't automatically solve this task, because the Semantic Web only promotes machine understandability of Web resources by explicitly providing a thick bunch of annotations, thus leaving their interpretation and use to the application. In this paper, we refer to such difficult-to-navigate open information systems as hyper-environments and we compare the interaction with a hyper-environment to a journey in which users are travelers and the aim of the journey is to find useful information.

We therefore propose a Semantic Navigation Engine that aims at helping Web travelers in traversing hyper-environments by giving them proper tools (called vehicles in the travel metaphor) that can help them in getting oriented and fulfilling their aims.
\end{abstract}

\section{Introduction}

The existing Web, the so-called syntactic Web, is a boundless environment in which Web users navigate, searching for useful information to achieve their goals.

Navigating in the syntactic Web, following links to move from a Web resource to another, has been compared (see [1], [2]) to the concept of travel in the physical world: a Web user acts like a traveler who tries to get oriented in an unfamiliar environment and often has to re-start and take different paths before finding the right way to the desired destination.

Adding a bit of semantics to the syntactic Web provides machines with a bunch of annotations, but we believe that this does not automatically imply that navigating the Semantic Web is any simpler.

In this paper, we name hyper-environment, in accordance with the travel metaphor, any open information system in which resources are described in a machine processable way and we introduce the concept of vehicle as the necessary tool to navigate effortlessly across a hyper-environment and to follow the most opportune path to reach the needed information.

We therefore propose a Semantic Navigation Engine which, building upon semantic annotations attached to resources, is able to provide users with proper 
vehicles, which support each part of their journey and suggest them the most suitable path to fulfil their specific tasks. In a few words, our Semantic Navigation Engine aims at providing end-users with multiple vehicles for traveling across hyper-environments.

In particular, for demonstrative purposes, we focus our attention on the medical general practice: if we apply the travel metaphor to this field, travelers are General Practitioners, the hyper-environment in which they move is a result set of a search on distributed and heterogeneous healthcare repositories, and their aim is to deepen their personal medical education in order to better manage their patient visits. In this paper we show how our Semantic Navigation Engine is able to provide General Practitioners with a customized vehicle that makes their travel across resources easier and more effective.

This paper is structured as follows: in section 2 we present the navigation problem and in section 3 the state-of-the-art approaches to solve it, both in the syntactic and in the Semantic Web fields; the concept of multiple vehicles to travel across hyper-environments is introduced in section 4, while the implementation of our Semantic Navigation Engine and our test-beds are described in sections 5 and 6 respectively.

\section{Getting Lost in Hyper-environments}

The fact that traveling across hyper-environments is possible does not mean that navigation is also easy or obvious to be undertaken. Below, we present the problem setting together with a possible usage scenario in the medical general practice.

\subsection{The Problem}

In the early days of the Web the lack of navigation plainness was considered as the navigation problem: users can get lost in a hyperspace and this means that, when users follow a sequence of links, they tend to become disoriented in terms of the goal of their original query and in terms of the relevance to their query of the information they are currently browsing [3]. The navigation problem has been long investigated in the hypermedia community and in particular some proposals tried to use the physical world as a model (e.g. Dillon in [2], Darken in [1]).

Following the physical world's metaphor, we notice that getting lost in a hypertext can be compared to getting lost in an unstructured space like a wood. In a structured space, for example when you get lost in a city, you can get oriented because streets have names and you just need to figure out your position on the two-dimensional structure of the city's map. On the contrary, when you get lost in a wood, you don't know where you are, how to reach your destination, and, often, you don't even know how to return to your original place. In a hypertext, like in a wood, you have to remember many trails and the way in which they are interconnected because most of them look just the same. As Nielsen writes in 
his famous book "Designing Web Usability: The Practice of Simplicity" [3], the navigation problem is still the unresolved problem in Web site usability.

The current effort to add semantics to the Web suggests augmenting userintended resources with machine-readable information, by means of metadata defined by ontologies. Considering also that all this additional information is provided with re-use in mind (thus authors are invited to put as much information as possible), the topology of the resulting hyper-text is much more complex than the already intricate topology of the Web. This is the reason why we name it a hyper-environment.

We believe that addressing the navigation problem in a hyper-environment is challenging but feasible, because semantic annotations provide machines with the ability to access what readers normally consider shared contextual information together with the information which is hidden in the resource.

\subsection{A Usage Scenario in the Medical General Practice}

General Practitioners are end-users that cannot afford to get lost. They are the category of physicians which is more exposed to medical errors. If they were well-informed about each novelty concerning diagnostics or treatments in the healthcare sector, they could considerably reduce their patients' risks. But being up-to-date is often a hard task, and probably it's impossible for General Practitioners to be informed about every single human pathology, so they generally prefer to prescribe an additional examination or to refer the patient to a specialist for a visit. They would spend time to deepen their medical knowledge only if they could be sure to reach easily and effortlessly the most suitable information, for example if they could come directly to the appropriate clinical guideline for a given pathology and access information regarding the available medical services, technologies and medications, their efficiency and side effects, possibly even relevant case studies or some specialist advice.

The following usage scenario describes the interaction between the end user (i.e. the General Practitioner) and the Semantic-based Healthcare Information Portal (named SHIP) we are conceiving in the COCOON project ${ }^{1}$, which provides a uniform, single point of access to heterogeneous and highly distributed medical information sources. This information may include articles from medical journals, scientific publications from specialized search engines and university libraries, electronically available clinical guidelines, as well as free text documents provided by each regional public health authority to local General Practitioners:

1. Geena, a General Practitioner, has come across an article in the British Medical Journal mentioning new breast cancer symptoms apparently discovered in the population of female smokers over the age of 40 . Intrigued by the article, and having relevant population among her patients, Geena decides to use SHIP, in order to collect additional information regarding this topic which may be useful in her future practice.

$\overline{1}$ http://www. cocoon-health.com 
2. Geena submits a query on early detection of breast cancer and an overwhelming amount of documents, together with their semantic annotations, is retrieved, but SHIP hides them behind a multifaceted display of the search results that includes a ranked search results list, relevant ontology concepts and documents associated with them. For each document SHIP provides the title, the publication date, the relevant keywords and a short excerpt.

3. Geena inspects the search results. She finds a document that she considers useful for her practice and follows the hyperlink to the original document text. SHIP offers her not only the document, but also hyperlinks to other documents thematically related to the selected one and the keywords used to semantically describe the document.

4. Geena follows the link to a keyword and a small bunch of related terminology is presented. She finds in this terminology a more specific term she did not think of when submitting the query, so she follows its hyperlink. SHIP provides her again with a small bunch of the terms and with links to the documents in the result set that are labeled with such keywords. This time Geena does not have to look for other documents, because she finds what she was looking for.

\section{$3 \quad$ State of the Art and Trends}

Over the last few years, many different research trends have tried to solve the navigation problem. We briefly summarize some of the most relevant ones, in order to frame the background of our solution.

\subsection{Syntactic Web Approaches}

Among the approaches which try to solve the navigation problem in the syntactic Web, an important position is occupied by trail engines. A trail engine $e^{2}$ is a sort of search engine, but it differs from a standard one because, in reply to a query expressed as a conjunction of keywords, a search engine returns a ranked list of pages (possibly containing all the keywords), whereas a trail engine returns a graph of pages connected by links, normally named trail (so that the user is adviced to follow a trail across a set of interconnected pages that represents a path via hyperlinks among the requested keywords).

The idea behind many trail engines is to model the Web as a network of nodes labeled with keywords. The Best-Trail Algorithm [4] models the Web via a finite automaton called Hypertext Finite Automaton (HFA). The states of a HFA represent Web pages, while transitions represent links. In this way, in a HFA, a valid trail leads the user to follow existing links from one node to the others in the trail. A slightly more complex way to model the Web was proposed in [5]. It consists in extending HFA by attaching probabilities to state transitions. These

$\overline{2}$ see e.g. http://trails.navigationzone.com/ 
probabilities can denote either the result of frequent user behavior patterns or the result of some calculation (e.g. average) over the relevancies of the pages in the trail.

In short, trail engines provide an affordable way to search for a trail in a set of already interconnected Web resources. In a way they try to cope with the lack of explicit semantics in the Web using models of the user behaviors that are supposed to partially capture what each page is about. Unfortunately, the known approaches don't seem to scale up.

\subsection{WWW Conceptual Model Approaches}

An alternative category of solutions to trail engines is provided by World Wide Web Conceptual Modeling approaches, or shortly the WWWCM approaches. They are characterized by the common goal of modeling a Web application at the conceptual level, in order to automatically implement it. Some successful attempts, such as WebML [6], W2000 [7], OOHDM [8], belong to this category. They show that a data-intensive Web application can be easily developed by separately modeling the domain information space, the navigation, the access and, in recent attempts, also the operations.

In the attempt to sketch out the least common denominator among the cited WWWCM approaches, we formulate the following definitions we will refer to in the rest of the paper:

- The domain information model describes the organization of the information managed by the Web application, in terms of the pieces of content that constitute its information base and of their semantic relationships. The schema of this model provides a shared understanding of the Web content that does not change or only changes very slowly over the time.

- The navigation models concern the facilities for moving across the application content; they represent the heterogeneous inter and intra-object navigation facilities the users can employ in traversing the information space of the Web application. These models are not necessarily shared among all users, but they are jointly employed by homogeneous categories of users.

- The access models concern the facilities for accessing information, i.e. the available access paths to objects in the information space. Access models specify the way in which the information described by the domain model is accessed: multiple access-models can be attached to the same domain information model, in order to specify different access semantics for different purposes. Each access model consists of collections of not strictly homogeneous objects.

Summing up, the WWWCM approaches are typically top-down, therefore they provide excellent solutions to manage the life cycle of a complex Web application, but this is mainly done on the strong assumption that all the information is under the control of the organization responsible for developing the Web application and that such application can be built from scratch. So, we might 
make to the WWWCM approaches the criticism that they address, and probably solve, the navigation problem in a closed context, but they do not address the navigation problem on the open Web. In a way, they break the principle according to which the Web is to be a universal, hence open, system.

\subsection{Semantic Web Approaches}

The lack of explicit semantics in the Web was perceived as an addressable problem by the knowledge representation community in the late '90s. SHOE [9] and OntoBroker [10] are successful attempts to show that such added semantics could prove to be very useful in solving the navigation problem. The idea is to define the terminology top-down (through ontologies) and use it bottom-up to annotate and wrap Web documents.

We believe that, nowadays, the major trend in solving the navigation problem is represented by the approaches based on the Semantic Web, whose common principle can be named ontology-supported and ontology-driven conceptual navigation. According to this principle, resources and links should be considered separately. Resources are self-contained items whose content can even be difficult to process automatically. Links are machine-processable descriptions (known as metadata) of the resources. These descriptions are provided by the authors in accordance with the terms described in one or more ontologies. In this way, every resource lies in a context made of semantic descriptions of terms and other resources that a machine can access and process. So the common situation experienced in the Web, whereby a reader can get lost if the author's intention does not match with the reader's intention, might be less common in the Semantic Web, because authors provide reusable descriptions while machines, being able to manage these descriptions, can adapt the interface to meet the readers' intentions. Therefore, complex role-based and integrated navigation structures can be built bottom-up on the fly, as long as each resource is described by the terms defined by shared ontologies, provided top-down.

Below, we provide a short description of some of the leading efforts in the Semantic Web approach:

- COHSE - a Conceptual Open Hypermedia Service [11] is an ontological reasoning service and Web-based open hypermedia link service integrated to form a conceptual hypermedia system, to enable documents to be linked via metadata describing their contents;

- SEAL (SEmantic portAL) and SEAL-II [12] show how ontologies can power information retrieval, greatly contributing to the combined goals of low-effort information integration and user-friendly information presentation;

- OntoWebber [13] supports the creation of reusable specifications of Web sites, by explicitly modeling the Web site via ontologies and employing semistructured data technology for data integration;

- ODESEW [14] is a rapid development tool for building ontology-based Web portals, which allows to configure the visualization of ontology-based information for different kinds of users; 
- SOIP-F [15] describes a framework for developing Organization Information Portals that deal with a small-scaled organizational Semantic Web, where resources are augmented with semantic annotations.

- OntoViews [16] is a Semantic Web portal tool for publishing RDF content on the Web; it combines the multi-facet search paradigm, developed within the information retrieval research community, with Semantic Web RDFS ontologies and extends the search service with a Semantic browsing facility based on ontological reasoning.

\section{Our Concept: Multiple Vehicles for Traveling Across Hyper-environments}

In this section we introduce our approach to the navigation problem, using a vivid metaphor to explain how users move across hyper-environments and how we can support and facilitate their navigation.

\subsection{The Travel Metaphor}

We believe that the physical world metaphor eases the problem of conceiving an open solution to the navigation problem, because users in traversing the hyperspace need the sort of information which is normally required to traverse the physical space. According to [17], users require the following information:

- Orientation information, necessary to find one's place within a body of interlinked resources. In designing a hypertext, one should therefore give an appropriate answer to the question "What can be done to orient users and help them to navigate efficiently and pleasantly?"

- Navigation information, necessary to make one's way through resources. In designing a hypertext, one should therefore give an appropriate answer to the question "While accessing a particular resource, how can users be informed about where the links related to that resource lead?"

- Exit or departure information, necessary to inform the users that they are leaving a given context. In designing a hypertext, one should therefore give an appropriate answer to the question "How can users retrace their steps in their going-on path?"

- Arrival or entrance information, necessary to inform the users that they are entering a given context. In designing a hypertext, one should therefore give an appropriate answer to the question "While accessing a new resource, how can users be assisted to feel "at home" in the new context?"

Goble et al. in [18] have moved further in the direction of introducing the notion of travel and mobility on the Web, to improve the accessibility of using the physical world as a model. They define travel as the confident navigation and orientation with purpose, ease and accuracy within an environment. This means that they extend navigation to include orientation, environment, mobility and purpose of the journey: 
- Orientation is the knowledge of the basic relationships between objects within the environment, and between the objects and the traveler.

- Environment is the context which the traveler traverses and includes the way in which the landscape is rendered and perceived.

- Mobility is the ease and confidence at which travel can be accomplished.

- The purpose of the journey is the reason why the traveler has chosen to undertake the journey.

Following this trend, Yesilada, Stevens and Goble introduce in [19] the concept of travel objects in order to describe how the hypertext environment is rendered and perceived by the travelers. In fact, travelers use or may need to use such environmental features or elements in order to make a successful journey, meaning that, when following a trail of information, they need to keep a sense of direction and they need a high mobility in terms of the goal of their original query and of the relevance to the query of the information they are currently browsing.

\subsection{The Vehicle Metaphor}

When we browse the Web, we are already accustomed to following links to move from one page to another. But, simply carrying out the action of clicking on a hyperlink, not only do we go ahead in our travel across Web resources, but we also make a decision about the direction of our trail in order to reach the most relevant information we are looking for. Furthermore, in different navigations, we follow different paths, either because we are looking for different information or because our task is more specific or more generic, so the granularity of the information we require is narrower or broader.

Following the travel metaphor, in this paper we introduce the idea that Web users need different vehicles to travel across resources on different occasions. A vehicle must support user navigation, suggesting the most relevant trail among all the possible paths a user can undertake, according to the purpose of the journey itself. A vehicle must provide valuable information about the environment, enabling orientation and supporting mobility in order to reach easily and effortlessly the travel destination. Moreover, in order to make his/her journey useful, a user needs the most appropriate vehicle to travel across the environment to achieve the specific purpose of his/her journey. Thus, he/she will request different vehicles that satisfy different needs not only during different travels, but also within the same journey: for example, when a Web traveler enters a hyperenvironment for the first time, he needs at the beginning a vehicle that helps him to get oriented and understand the spatial relationships among the resources; afterwards, when he/she feels familiar within the new environment, the same traveler needs another vehicle to move on in his/her journey, to deepen his/her knowledge about a particular section of that hyperspace, looking for more detailed information.

What users need in their navigation is the most appropriate view on resources, meaning that, in every step of their travel, they don't need all the available information, but only a part of it, the part related to the information they are looking for. 
Thus, when conceiving the vehicle metaphor, we asked ourselves what kind of view on resources a vehicle must provide the user with. To answer this question in the most precise and effective way, we noticed that in our everyday experience of navigation through the Web, we can look at the term view under, at least, two different aspects; we can therefore give two different meanings to a view on a particular resource:

1. View as presentation of a subset of all the available information regarding the resource; if we divide all the knowledge about a particular item in travel objects, i.e. atomic bunches of information, we can build a view by composing together these elementary "bricks"; in this meaning, two views on the same resource differ in the set of travel objects employed in giving information about a resource (a generic description instead of a detailed presentation, a certain set of aspects or features instead of another, and so on).

2. View as navigation from that resource to another one following hyperlinks; the view can suggest different paths to cross information, which can be related to the particular phase of the travel; in this meaning, two views on the same resource differ in the possible directions they suggest the user for the continuation of his/her journey.

\section{Our Implementation}

Our work tries to solve the navigation problem building vehicles to support the users' journey across resources and building upon semantics attached to resources in order to make traveling significant and effective to attain the users' tasks.

Starting from an early prototype we already described in [15], we refine the browsing-time support of SOIP-F, a framework that supports the building of Semantic Organizational Information Portals, introducing the Semantic Navigation Engine by adding a presentation model, including a way to handle travel objects and a simple way to describe a model in term of navigation, access and presentation models. As a result we obtain the more powerful version of SOIP-F we describe below.

\subsection{A Technical Overview of SOIP-F}

At first glance, SOIP-F might appear as a radical new departure in Web portal design, but it is not. SOIP-F is implemented bringing together existing and well understood technologies:

- it uses a Web Framework that implements the well-known Model-ViewController (MVC) design pattern,

- it follows the $W W W$ Conceptual Model approach in separately modeling domain information space, navigation, access and presentation,

- it requires portal administrators to specify conceptual models using ontologies written in OWL-DL, 
- it manages and stores RDF encoded metadata that describe the resources in a machine processable way.

An important requirement taken in consideration during the design of SOIP-F is the strong decoupling between the portal and the information sources, because the aim of an organization portal is to provide a single and user-tailored point of access to all organizational content sources.

SOIP-F, in fact, takes from the WWWCM the idea of separately modeling domain information space, navigation, access and presentation. Following such an approach in modeling portals independently of the domain, SOIP-F proposes a portal ontology that includes portal-dependent terminology: structural terms such as entity or component, navigation terms such as contains or related_to, access terms such as next or down and presentation terms like title, text-box or image.

This strong requirement for decoupling between the portal and the information sources expects a portal built using SOIP-F to be just one of the many applications accessing content sources; for this reason, SOIP-F doesn't require the information to be structured in any particular way. So, differently from most WWWCM approaches, SOIP-F proposes to model navigation, access and presentation by mapping the domain terminology into the portal terminology, creating, in a bottom-up approach, a relation between domain-dependent terms and portal-dependent terminology.

In particular, as we anticipated, modeling a vehicle for our Semantic Navigation Engine in order to travel across a portal built using SOIP-F implies the operation of mapping, through the composition of navigation, access and presentation models. We explain the meaning of these models in SOIP-F as follows:

- The navigation model takes into account the possible paths across homogeneous resources (for example, it states which relationships must be underlined and emphasized); it can be shared among many users with similar aims.

- The access model takes into account the possible paths across heterogeneous resources which share some meaning (for example an access model can suggest an ordered list of resources to be navigated serially or a set of "most visited" or "recently added" pages); it is specific of the aim but it can be built by querying the domain information model.

- The presentation model takes into account the composition of different parts of information on the page, the order and the layout of the presentation (for example, a presentation model states number, type and position of the "boxes" that set up a page); it can include both shared travel objects (that are provided for general purposes) and user specific travel objects (that are provided to support the user in a specific part of his/her travel). 


\subsection{A Structural Overview of SOIP-F}

Our Semantic Navigation Engine builds upon the fact that SOIP-F is an extendable J2EE application framework that can be configured using a set of OWL ontologies.

The framework itself is not a portal, because it needs at least a domain ontology and a set of "content sources" to be crawled. Once this information is provided, the content sources become browsable using a low-level vehicle we name "metadata-vehicle". Starting from a resource, this vehicle shows in a table all the RDF triples that involve the selected resource. Each subject, property and object is provided as a link that the end-user can follow in order to select another resource.

In the same way, other vehicle descriptions can be provided. Each vehicle description includes a navigation model, an access model and a presentation model. In particular, the presentation model describes which travel objects are displayed for each type of resource. Each travel object is either responsible for presenting the information carried by some metadata or for providing a link the user can follow to move to other parts of the hyper-environment.

Each travel object is made up of two parts: a decorator and a template. A decorator is a Java class that is responsible for querying the reasoner and for extracting the information that the travel object will show, whereas a template describes the visual appearance of the information provided by the decorator.

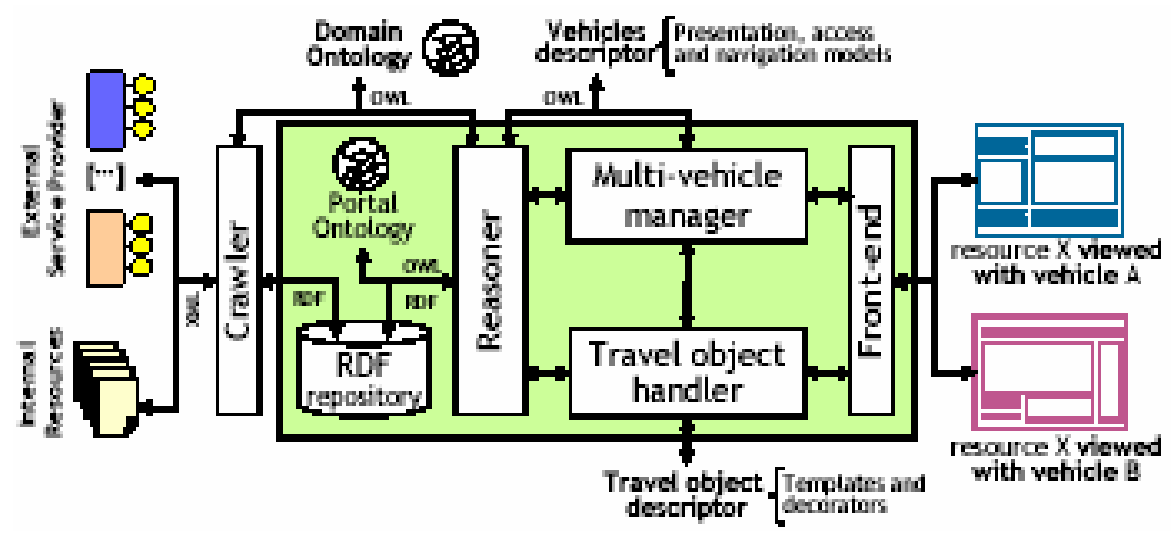

Fig. 1. SOIP-F structural overview

SOIP-F (see figure 1) is composed of five logical components:

- the reasoner, which manages both the terminologies and the assertions (except for literals). If no configuration information is provided, it only contains the portal ontology. It also offers query facilities for the other components. It is based on RACER ${ }^{3}$ and extends JRacer APIs;

\footnotetext{
${ }^{3}$ http://www.sts.tu-harburg.de/ r .f.moeller/racer/
} 
- the RDF repository, which stores the metadata describing the resources; it offers the reasoner query facilities for selecting literals and it offers the crawler query facilities for inserting, deleting and updating metadata. It is based on Jena framework ${ }^{4}$ and can use either the file system or a database as permanent storage facility.

- the multi-vehicle manager, which enables end-users to select a vehicle and/or to change it during the navigation. It uses the facilities provided by the reasoner in order to mount the most appropriate navigation, access and presentation model.

- the travel object handler, which uses, according to the current vehicle selected, the appropriate decorators to query the reasoner and the right templates to produce a HTML-based presentation of each travel object.

- the front-end, which is made up of several Java Servlets that share a Velocity template engine ${ }^{5}$. Together they implement a MVC design pattern in a J2EE environment.

These components can be extended with another one: during the building of a portal with SOIP-F, a crawler can be developed to extract data, properly annotated with metadata described by one or more domain-specific ontologies, from content sources and to make them available to be presented and navigated within the portal.

\section{Our Test-Beds}

To prove our approach we built some test-portals on the top of our SOIP framework (see http://seip.cefriel.it). We briefly propose the most significant one, which (tries to) solve the navigation problem in the healthcare domain, and then we present other test-implementations demonstrating our approach.

\subsection{Aiding General Practitioners in Navigating a Healthcare Hyper-environment}

As introduced in $\S 2.2$, in the COCOON project we have to satisfy the requirements of a General Practitioner who would like to be informed and up-to-date about every medical information that could be useful to better manage his/her visits, in order to provide the most suitable treatment or to prescribe the most useful examination to his/her patients.

A Semantic-based Healhcare Information Portal (SHIP, as abbreviated before) could be very useful during the general practitioner's search for information, supporting his/her queries, returning the most interesting results and suggesting different significant paths to navigate across the result set.

We built a prototype of SHIP on the top of our SOIP-framework, designing a portal to help general practitioners to travel, easily and effortlessly, in a

\footnotetext{
${ }^{4}$ http://jena.sourceforge.net/

${ }^{5}$ http://jakarta.apache.org/velocity/
} 
healthcare hyper-environment. We based our search facilities on the Web services provided by Entrez PubMed ${ }^{6}$, called e-utilities, that allow to search in PubMed, MeSH and other medical databases. We developed an ad-hoc component, an invoker that, querying Entrez e-utilities through pre-formatted queries (called search strategies), is able to retrieve information about medical articles; Entrez services return results in XML-format that our component translates, through an XSL transformation, to RDF-format described by a simple OWL ontology.

The result set is therefore made up of a set of resources described with metadata: each article presents a bunch of information about the document itself (such as authors, title, medical journal, abstract, the link to the actual document, etc.) together with cues about its semantics, i.e. the medical keywords that describe the meaning of the article. This terminology comes from $\mathrm{MeSH}^{7}$ (Medical Subject Headings), a thesaurus of medical terms that are semantically interconnected (for example, a term is linked to a broader or narrower one, or a term about a pathology is linked to the term of the affected bodypart); these terms can be exploited to put in relation articles sharing the same semantics.

Once the result set is available to be crawled and accessed via the portal, our Semantic Navigation engine presents the results to the final user, giving him/her a vehicle to travel across them. This vehicle is able to suggest more than one path to navigate through resources: besides the path that offers access to the results as in a ranked ordered list and that lets the user navigate from an article to the subsequent one just following a "next article" link, SHIP offers the possibility of navigating through resources following the shared keywords. While in the first case the General Practitioner just follows a list (even if the order of the retrieved documents can be altered to take into account the user's preferences and interests), in the latter case the final user can navigate through keywords as well as through documents and can exploit this information to better understand the retrieved results and, if necessary, to refine his/her search strategy using the most appropriate keywords to re-query Entrez databases.

Readers wishing to try SHIP can do so on http://seip.cefriel.it/ship.

\subsection{Other Demonstrative Portals}

Some other test portals that we built to demonstrate our approach are available on line. These demonstrative portals illustrate the possibilities and potentialities of our Semantic navigation Engine in SOIP-framework and we introduce them briefly.

Virtual Museum of Contemporary Art portal - it aggregates data (in Italian) about artworks and artists from different real museums, letting virtual visitors

\footnotetext{
${ }^{6}$ http://www.ncbi.nlm.nih.gov/Entrez/index.html

7 MeSH is a thesaurus developed by US National Library of Medicine since 1954; further information can be obtained in http://www.nlm.nih.gov/mesh/meshhome.html
} 
travel across resources with different vehicles: the portal offers a thematic trail vehicle (a guided tour across artworks of a particular artistic movement or period), but also a detailed trail vehicle (that allows to investigate the work of a particular artist).

Semantic Web Virtual Lesson - it's a portal built as a unifying view on different material taken from some presentations about fundamentals, technologies and applications of Semantic Web; it allows end-users to move from a presentation to another just following links to semantically related slides, as if it was a single lesson.

CEFRIEL organization portal - CEFRIEL's information about units, people, projects, research fields is enriched with semantic annotations that allow the Semantic Navigation Engine to present resources to different users in different ways: there are various views centered on units, on ongoing projects or on research fields.

\section{Conclusions}

Our proposal for a Semantic Navigation engine is a joint attempt to solve the navigation problem bringing together Semantic Web technologies, the WWWCM approach and various studies on users' habits and needs in browsing the Web. We identify the core problem in navigating through a hyper-environment with the difficulty of moving across different resources, maintaining a good sense of orientation and reaching the desired destination while covering a path that makes the user/traveler enrich his/her knowledge and attain his/her aim.

We believe that the key innovations introduced by our proposal for a Semantic Navigation engine are the following:

- loose coupling between domain information model (captured by the organizational ontology) and the various navigation, access and presentation models;

- bringing in the Semantic Web community the efforts of the WWWCM approach in defining a (top-down) terminology for navigation, access and presentation;

- building navigation, access and presentation models in an indirect way, by mapping domain information terminology to navigation, access and presentation terminology (as in a bottom-up approach).

To achieve this goal, our Semantic Navigation Engine builds upon the definition of vehicle as a composition of navigation, access and presentation models; for each Semantic Navigation Engine, we can define a set of vehicles. In fact, different vehicles might be useful in different parts of the hyper-environment, in the same way as real vehicles (bicycles, cars, trains, planes) are used to take different kinds of journeys in the real world. In this way, we make a step forward in the direction of uncoupling domain knowledge from the way to access it. 


\section{Acknowledgments}

The research has been partially supported by the COCOON Integrated Project (IST FP6-507126) while the implementation of SOIP-F has been partially funded by Engineering as part of the activities of the XVI Master in Information Technology of CEFRIEL - Politecnico of Milano. We thank Maurizio Brioschi, Stefano Ceri and Nahum Korda for their precious contributions.

\section{References}

1. R.P. Darken, J.L. Sibert: Wayfinding Strategies and Behaviors in Large Virtual Worlds. In: Proc ACM CHI'96. (1996) 142-149

2. A. Dillon, M.W. Vaughan: "It's the Journey \& the Destination": shape and the emergent property of genre in evaluating digital documents. The New Review of Hypermedia and Multimedia 3 (1997) 91-106

3. J. Nielsen: Designing Web Usability: The Practice of Simplicity. New Riders Publishing, Thousand Oaks, CA, USA (2000)

4. R. Wheeldon, M. Levene: The Best Trail Algorithm for Assisted Navigation of Web Sites. In: WWW2003, Budapest, Hungary. (2003)

5. M. Levene, G. Loizou: A Probabilistic Approach to Navigation in Hypertext. Information Sciences 114 (1999) 165-186

6. S. Ceri, P. Fraternali, A. Bongio: Web Modeling Language (WebML): a modeling language for designing Web sites. Computer Networks (Amsterdam, Netherlands: 1999) 33 (2000) 137-157

7. L. Baresi, F. Garzotto, P. Paolini, S. Valenti: HDM2000: The HDM Hypertext Design Model Revisited. Tech. report, Politecnico di Milano (Jan. 2000)

8. D. Schwabe, G. Rossi, S.D.J. Barbosa: Systematic hypermedia application design with OOHDM. In ACM, ed.: Hypertext '96, Washington, DC, March 16-20, 1996: the Seventh ACM Conference on Hypertext: Proceedings, New York, NY, USA, ACM Press (1996) 116-128

9. J. Heflin, J. Hendler, S. Luke: SHOE: A Blueprint for the Semantic Web. In: Spinning the Semantic Web. MIT Press, Cambridge, MA (2003)

10. D. Fensel, J. Angele, S. Decker, M. Erdmann, H.-P. Schnurr, S. Staab, R. Studer, A. Witt: On2broker: Semantic-based access to information sources at the WWW. In: WebNet (1). (1999) 366-371

11. L. Carr, W. Hall, S. Bechhofer, C.A. Goble: Conceptual linking: ontology-based open hypermedia. In: World Wide Web. (2001) 334-342

12. A. Hotho, A. Maedche, S. Staab, R. Studer: SEAL-II - The Soft Spot between Richly Structured Unstructured Knowledge. Journal of Universal Computer Science 7 (2001) 566-590

13. Y. Jin, S. Xu, S. Decker, G. Wiederhold: Managing Web Sites with OntoWebber. Lecture Notes in Computer Science 2287 (2002) 766

14. O. Corcho, A. Gomez-Perez, A. Lopez-Cima, V. Lopez-Garcia, M. Suarez-Figueroa: ODESeW. Automatic Generation of Knowledge Portals for Intranets and Extranets. In D. Fensel, ed.: The Semantic Web, ISWC 2003, LNCS 2870. (2003) 802-817

15. E. Della Valle, M. Brioschi: Toward a framework for Semantic Organizational Information Portal. In proceedings of first European Semantic Web Symposium, ESWS2004 (2004) 
16. E. Makelä, E. Hyvönen, S. Saarela, K. Viljanen: OntoView - A Tool for Creating Semantic Web Portals. In: Proceedings of the 3rd International Semantic Web Conference (ISWC 2004), Hiroshima, Japan (2004)

17. G.P. Landow: Hypertext 2.0: The Convergence of Contemporary Critical Theory and Technology. Johns Hopkins University Press (1997)

18. C.A. Goble, S. Harper, R. Stevens: The travails of visually impaired web travellers. In: UK Conference on Hypertext. (2000) 1-10

19. Y. Yesilada, R. Stevens, C. Goble: A Foundation for Tool Based Mobility Support for Visually Impaired Web Users. In: Proceedings of the Twelfth International World Wide Web Conference. (2003) 